\title{
Comparison of safety, effectiveness and serum inflammatory factor indexes of Saccharomyces boulardii versus Bifidobacterium triple viable in treating children with chronic diarrhea: a randomized trial
}

\author{
Jiawei Zhang ${ }^{1}$, Su Wan ${ }^{2}$, Qingqing Gui ${ }^{1}$ \\ ${ }^{1}$ Pharmacy Department, Zhongda Hospital Affiliated to Southeast University, Nanjing, China; ${ }^{2}$ Pharmacy Department, Nanjing Liuhe Renming \\ Hospital, Nanjing, China \\ Contributions: (I) Conception and design: J Zhang; (II) Administrative support: S Wan; (III) Provision of study materials or patients: Q Gui; (IV) \\ Collection and assembly of data: J Zhang; (V) Data analysis and interpretation: All authors; (VI) Manuscript writing: All authors; (VII) Final approval \\ of manuscript: All authors. \\ Correspondence to: Jiawei Zhang. Pharmacy Department, Zhongda Hospital Affiliated to Southeast University, Nanjing 210009 , China. \\ Email: 439953514@qq.com.
}

Background: Diarrhea is common in children under 5 years of age and is an important public health problem in China. CD is the main obstacle to the growth and development of children, which brings a great burden to individuals, families and society. The objective of this work is to study the efficacy and safety of Saccharomyces boulardii versus Bifidobacterium triple viable in the treatment of CD in children.

Methods: From October 2018 to October 2020, a total of 161 children aged 2-8 years hospitalized with CD were randomly allocated into $S$. boulardii group, Bifidobacterium triple viable group and control group. After 14 days of treatment, the curative effect and recovery time of the three groups were evaluated. The levels of serum interleukin (IL)-6, IL-7 and tumor necrosis factor-alpha (TNF- $\alpha$ ) before and after the treatment were valuated and compared among the three groups, together with clinical efficacy and safety.

Results: The recovery time of the Bifidobacterium triple viable group was significantly shorter than that of $S$. boulardii group $(\mathrm{P}<0.05)$. The marked effective rate and total effective rate of the Bifidobacterium triple viable group were significantly higher than those of the control group $(\mathrm{P}<0.05)$; the total effective rate of the $S$. boulardii group was significantly higher than that of the control group $(\mathrm{P}<0.05)$. The improvement in the levels of IL-6, IL-7 and TNF- $\alpha$ in the Bifidobacterium triple viable group was greater than that in the control group; the improvement in IL-6 and IL-7 levels in the Bifidobacterium triple viable group was greater than that in the S. boulardii group; the improvement in IL-6 and IL-7 levels in the S. boulardii group was greater than that in the control group, and the differences were statistically significant $(\mathrm{P}<0.05)$

Conclusions: The efficacy of Bifidobacterium triple viable and S. boulardii in the treatment of children with $\mathrm{CD}$ was better than that of conventional treatment. The treatment effect for Bifidobacterium triple viable was more significant, and it was proved to be safe, to shorten the course of disease, and have clinical relevance.

Trial registration: Chinese Clinical Trial Registry ChiCTR2100046444.

Keywords: Bifidobacterium triple viable; children; chronic diarrhea (CD); Saccharomyces boulardii; serum inflammatory factors

Submitted Apr 08, 2021. Accepted for publication Jun 01, 2021.

doi: $10.21037 / \mathrm{tp}-21-195$

View this article at: http://dx.doi.org/10.21037/tp-21-195

(C) Translational Pediatrics. All rights reserved. 


\section{Introduction}

Diarrhea is one of the most important public health problems worldwide. It refers to a gastrointestinal syndrome caused by multiple factors, but characterized by increased frequency of stools and a change in stool characteristics. Diarrhea mainly affects children under 5 years old, especially infants under 2 years old. According to the World Health Organization report in 2017, there are $\sim 1.7$ billion cases of diarrhea in children worldwide every year, and $\sim 52,5000$ children aged under 5 years die as a result (1). Diarrhea is classified according to the course of disease and can be divided into: acute diarrhea: course of disease $<2$ weeks; persistent diarrhea: course of disease between 2 weeks to 2 months; chronic diarrhea: course of disease $>2$ months (2). Diarrhea can cause malnutrition, growth retardation and cognitive impairment in children, and malnutrition can aggravate diarrhea and affect its recovery, thus forming a vicious circle (3). In recent years, with the improvement of living standards and the use of oral rehydration salts, the incidence and mortality of children with acute diarrhea have been significantly reduced. However, the incidence of chronic diarrhea is still high due to complex etiology and difficult treatment, and the patients are mainly children under 5 years old, which seriously affects the growth and development of children. Chronic diarrhea (CD) is generally defined as diarrhea lasting at least 2 weeks (4), and its etiology can be divided into four pathophysiological mechanisms: osmotic, secretory, dysmotility-associated and inflammatory (5).

The etiology of CD in children is complex, because is related to age, diet, climate, living environment, socioeconomic development and genetic factors. In addition to acquired factors, infants can have congenital anatomical abnormalities and congenital genetic diseases. Infection is still the main cause of CD in children, but with improvements in health conditions, the incidence rate of allergic diseases is increasing. The industrialized development of society, tremendous learning pressure and fast pace of life has made the incidence rate of inflammatory bowel disease increase in children.

Saccharomyces boulardii is a nonpathogenic fungal probiotic, which evidence-based medicine has proved has a clear effect on the treatment of acute infection, nonspecific diarrhea, antibiotic-associated diarrhea (AAD) and irritable bowel syndrome (6). S. boulardii participates in the maintenance of the intestinal microbial barrier by inhibiting pathogenic bacteria, antagonizing the colonizing function, and stimulating intestinal immune and nutritional functions. Moreover, S. boulardii can survive in the digestive system for a long time, and is not be destroyed by gastric acid, bile acid or antibiotics. S. boulardii can also activate the reticuloendothelial system and complement system; increase the secretion of human intestinal immunoglobulin (S-IgA); activate the activity of zincbinding metalloproteinase; improve the secretion of host disaccharidase; improve the absorption function of the host; inhibit the growth and reproduction of intestinal pathogenic microorganisms and the adhesion and invasion of mucosal epithelial cells; release low-molecular-weight protease to neutralize, inactivate and degrade bacterial toxins; alleviate abnormal intestinal secretion;, inhibit the translocation of nuclear factor-kappa B (NF- $\kappa \mathrm{B})$ in the inflammatory signaling pathway; and inhibit the inflammatory signaling pathway, thus playing an anti-inflammatory role $(7,8)$.

Bifidobacterium triple viable bacteria comprises Bifidobacterium, Lactobacillus and Enterococcus to directly supplement the beneficial flora of children's intestines, help restore the normal flora distribution in children, inhibit the growth of pathogenic bacteria, and reduce the absorption of endotoxin by intestinal mucosa (9). Because the intestinal flora is an integral part of the intestinal mucosal barrier, the normal distribution of intestinal flora is also conducive to the recovery of intestinal mucosal barrier function in children (10). Clinical studies have shown that normal intestinal flora can also promote the absorption of nutrients, synthesize vitamins needed for growth and development, and improve immune function, which is conducive to the treatment and rehabilitation of infectious diseases (11). If diarrhea is not treated in time, it can lead to acid-base imbalance, water electrolyte disorder, and even be life-threatening in children (12). Both S. boulardii and Bifidobacterium are nonpathogenic fungal probiotics that can regulate immune function and intestinal flora, improve host absorption function, alleviate abnormal intestinal secretion, and have anti-inflammatory and antiviral effects. Wang et al. (13) found that treatment of acute diarrhea in children with Saccharomyces boulardii combined with Bifidobacterium can effectively shorten the duration of diarrhea and hospital stay, reduce the number of diarrhea and enhance the cellular immune function. However, they are rarely used in children with CD $(14,15)$. The purpose of this study was to compare the efficacy of $S$. boulardii and Bifidobacterium triple viable in the treatment of children with $\mathrm{CD}$, in order to find an effective, stable and safe microecological treatment, and 


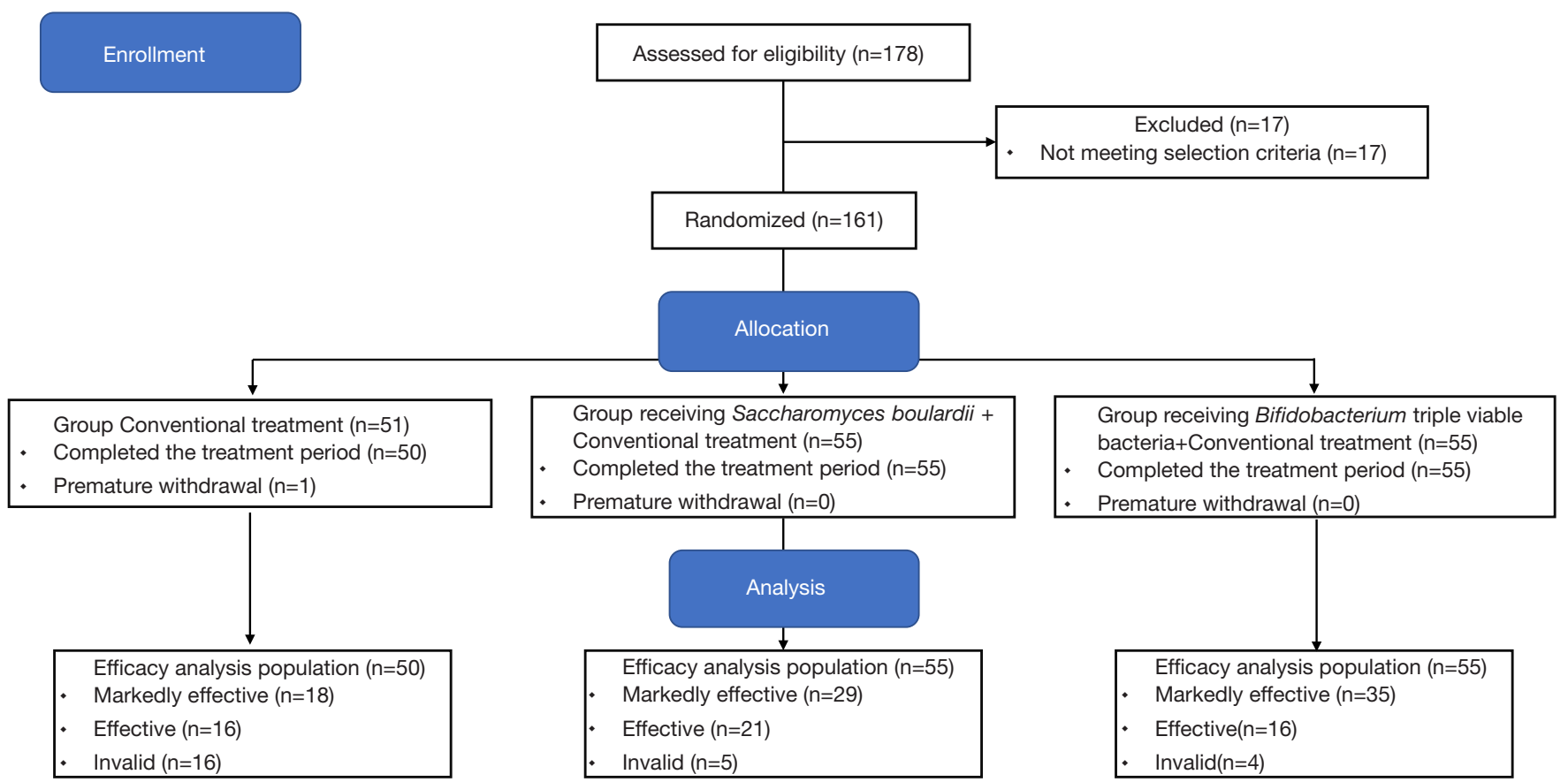

Figure 1 Flowchart of participants through the study of Saccharomyces boulardii vs. Bifidobacterium triple viable bacteria in treatment of chronic diarrhea in children.

provide a reference for the clinical treatment of children with CD.

We present the following article in accordance with the CONSORT reporting checklist (available at http://dx.doi. org/10.21037/tp-21-195).

\section{Methods}

\section{Setting and study design}

This study included 161 children (age, 2-8 years) with CD who were hospitalized in Zhongda Hospital from October 2018 to October 2020. The children were first numbered according to their order of admission, and then divided into three groups using the random number table method by a research physician: $S$. boulardii group (conventional treatment $+S$. boulardii, $\mathrm{n}=55$ ), Bifidobacterium triple viable group (conventional treatment + Bifidobacteria triple live bacteria, $n=55$ ), and control group (conventional treatment, $\mathrm{n}=51)$. We observed and recorded the recovery time, curative effect, serum interleukin (IL)-6, IL-17, and tumor necrosis factor-alpha (TNF- $\alpha$ ) indicators before and after treatment, and the occurrence of adverse reactions in the three groups of children after 14 days, as well as clinical treatment effect and safety. The parents of all study subjects who met the criteria for inclusion gave informed consent. The process is shown in Figure 1.

Inclusive criteria: (I) defecation frequency $\geq 3$ times/day and fecal characteristics in accordance with Bristol fecal characteristics types 6 and 7; (II) duration of diarrhea symptoms $\geq 2$ weeks; (III) age 2-8 years; (IV) routine fecal examination without white or red blood cell counts.

Exclusion criteria: (I) mucus stool or pyorrhea; (II) had taken other probiotics, antidiarrheal drugs or drugs affecting gastrointestinal motility within 2 weeks before inclusion in this study; (III) history of allergic reaction to probiotics.

This study was conducted in accordance with the ethical principles of the Declaration of Helsinki (as revised in 2013), and has been approved by the ethics committee of Zhongda Hospital Affiliated to Southeast University (No. 20181092).

\section{Medical history}

Demographic and medical history data, such as sex, age, height, weight, course of disease, etc. were collected at the time of enrollment. The number of bowel movements and stool characteristics in the week prior to consultation were 
recorded as baseline data, and we performed routine stool, blood, and liver and kidney function tests. We also recorded the pretreatment levels of serum IL-6, IL-17 and TNF- $\alpha$. After 2 weeks of treatment, we again recorded the number of bowel movements and stool characteristics, adverse reactions, and rechecked routine blood, liver and kidney function tests, and serum IL-6, IL-17, and TNF- $\alpha$ levels.

\section{Treatments}

The control group was given routine treatments such as oral montmorillonite powder, rehydration salt and intravenous rehydration, while the $S$. boulardii group was given routine treatment and oral $S$. boulardii [Biocodex (France) $0.25 \mathrm{~g} \times 6$ bags, batch number: s20150051], and the dosage was selected according to the age of the children as follows. Children $>3$ years old: one bag twice daily; children <3 years old: one bag once daily. The Bifidobacterium triple viable group was given conventional treatment and oral Bifidobacterium triple viable capsules (Shanghai Xinyi Pharmaceutical Co., Ltd. specification: each capsule contains $210 \mathrm{mg}$ powder, batch No. Guoyao Zhunzi s10950032). The dosage was selected according to age: children $>3$ years old, 2 capsules each time, twice daily; children $<3$ years old, $1-2$ capsules each time, twice daily. The powder in the capsule could be administered in warm boiled water. The course of each treatment was 14 days.

\section{Serum inflammatory factors}

Before treatment and 14 days after treatment, $5 \mathrm{~mL}$ sample of peripheral venous blood from the forearm of each child was drawn, centrifuged at $1,000 \mathrm{~g}$ for $15 \mathrm{~min}$, removal of the supernatant for storage at $-80{ }^{\circ} \mathrm{C}$ before testing for changes in serum IL-6, IL-17, and TNF- $\alpha$ levels using a double antibody sandwich enzyme-linked immunosorbent assay [Elabscience (catalog Nos. E-ELN-H0102c, E-ELH0105c, E- EL-H0109c]. All tests were performed in strict accordance with the manufacturers' instructions.

\section{Efficacy evaluation}

According to the Chinese Diarrhea Disease Diagnosis and Treatment Program (16), the curative effect of CD is divided into three types: markedly effective [number of stools returned to normal ( $\leq 2$ times/day) and stool characteristics returned to normal (Bristol types 3, 4, 5)]; effective [defecation frequency returned to normal after treatment ( $\leq 2$ times/day) or stool characteristics returned to normal (Bristol types 3, 4, 5]; ineffective, defecation frequency still $\geq 3$ times/day after treatment, and stool characteristics still Bristol classification 6 and 7. The total effective rate $(\%)=$ (number of markedly effective cases + number of effective cases)/total number of people $\times 100 \%$. The recovery time was recorded, and the final evaluation of efficacy was performed on the 14th day of treatment.

\section{Adverse reaction registration}

Any adverse reactions during the treatment of all patients were recorded and compared with the blood routine and liver and kidney function tests before treatment.

\section{Statistical analysis}

SPSS 22.0 software was used for statistical analysis. The measurement data with a normal distribution was expressed by $\bar{x}_{ \pm \mathrm{s}}$. The paired $t$-test was used for comparison before and after treatment in the same group, and the $t$-test of independent samples was used for comparison between groups. Chi-square test was used for comparison of count data between groups. $\mathrm{P}<0.05$ indicated a statistically significant difference.

\section{Results}

\section{Baseline data}

A total of 161 patients with $\mathrm{CD}$ of various etiologies were included. The main causes were allergic diarrhea (51/161, $31.68 \%)$, intestinal infection $(71 / 161,44.10 \%)$, lactose intolerance (21/161, 13.04\%) and AAD (26/161, 16.15\%); 49 cases $(30.43 \%)$ had unclear etiology and 112 cases (69.57\%) had clear etiology. Among the children with a clear cause, 36 cases were caused by single factor $(22.36 \%)$, and 76 cases were caused by multiple factors $(47.20 \%)$. Among them, allergic diarrhea combined with intestinal infection, intestinal infection combined with lactose intolerance, and AAD mainly associated with intestinal infection. Baseline characteristics of the study population are presented in Table 1. There were no significant differences in serum IL-6, IL-17, and TNF- $\alpha$ levels among children with $\mathrm{CD}$ before treatment $(\mathrm{P}>0.05)$. The process is shown in Figure 1. 
Table 1 Baseline characteristics of participants: comparison of treatment groups

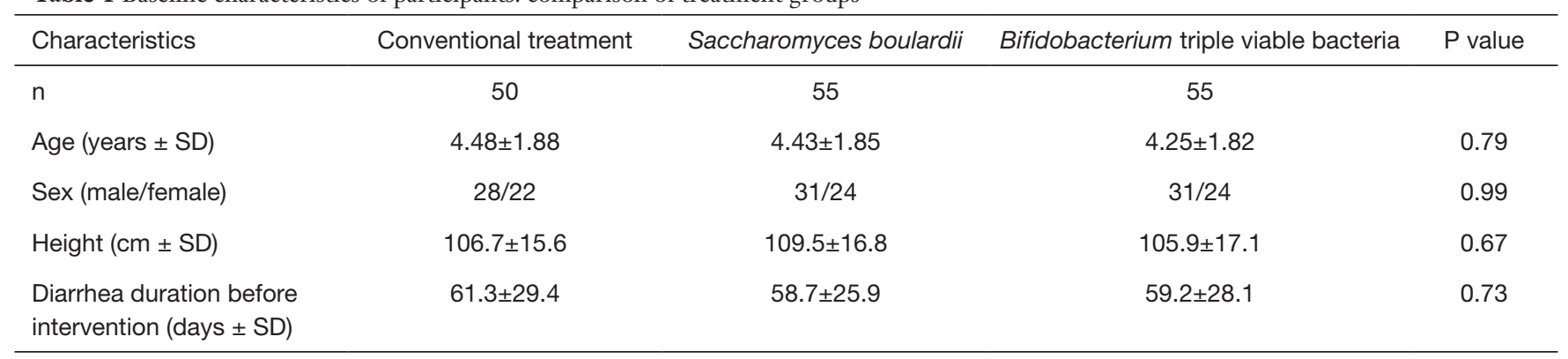

Table 2 Comparison of efficacy after treatment of patients in three groups

\begin{tabular}{lccccc}
\hline Group & Cases $(\mathrm{n})$ & Markedly effective, $\mathrm{n}(\%)$ & Effective, $\mathrm{n}(\%)$ & Invalid, $\mathrm{n}(\%)$ & Total effective, $\mathrm{n}(\%)$ \\
\hline Control group & 50 & $18(22.0)^{\#}$ & $16(30.2)$ & $16(30.2)^{\star \#}$ & $34(68.0)^{\star \#}$ \\
S Saccharomyces boulardii group & 55 & $29(35.4)$ & $21(39.6)$ & $5(9.1)$ & $50(90.9)$ \\
Bifidobacterium triple viable group & 55 & $35(42.7)$ & $16(30.2)$ & $4(7.3)$ & $51(92.7)$ \\
\hline
\end{tabular}

*, $\mathrm{P}<0.05$ vs. the effect of $\mathrm{S}$. boulardii group. ${ }^{\#}, \mathrm{P}<0.05$ vs. the effect of Bifidobacterium triple viable group.

\section{Comparison of curative effect}

In the control group after treatment, a total of 26 returned to normal defecation frequency ( $\leq 2$ times/day), 26 had stool properties return to normal, and in 18 cases both returned to normal at the same time, giving an effective rate of $30.2 \%(16 / 50)$, and a markedly effective rate of $22.0 \%$ $(18 / 50)$; in the S. boulardii group, there were 39 cases of defecation frequency returning to normal after treatment ( $\leq 2$ times/day), 40 cases of stool characteristics returning to normal, 29 cases of both returning to normal at the same time, giving an effective rate of $39.6 \%$ (21/55), and markedly effective of $35.4 \%$ (29/55); after treatment with Bifidobacterium triple viable bacteria, there were 43 cases of defecation frequency returning to normal ( $\leq 2$ times/day), 43 cases of stool characteristics returning to normal, and 35 cases of both recovering to normal at the same time, giving an effective rate of $30.2 \%(16 / 55)$, and a markedly effective of $42.7 \%(35 / 55)$.

There was a significant difference between the control and Bifidobacterium triple viable bacteria groups after treatment and the total effective rate $(\mathrm{P}<0.05)$; the control and $S$. boulardii groups showed a significant difference after treatment $(\mathrm{P}<0.05)($ Table 2).

\section{Comparison of recovery time}

The recovery time of the Bifidobacterium triple viable group was significantly shorter than that of the S. boulardii and control groups, the recovery time of the $S$. boulardii group was significantly shorter than that of the control group, and the difference was statistically significant $(\mathrm{P}<0.05)$ (Figure 2, Table 3).

\section{Levels of IL-6, IL-17 and TNF- $\alpha$ before and after treatment}

There was no significant difference in IL-6, IL-17, and TNF- $\alpha$ among the three groups of children before treatment $(\mathrm{P}>0.05)$, but after the treatment, all the indicators improved, and the degree of improvement in IL-6, IL-17 and TNF- $\alpha$ levels in the Bifidobacterium triple viable group was better than in the control group (statistically significant difference: $\mathrm{P}<0.05$ ); the improvement in IL- 6 and IL17 levels in the Bifidobacterium triple viable group was better than in the $S$. boulardii group (statistically significant difference: $\mathrm{P}<0.05$ ); the improvement in IL-6 and IL-17 levels in the S. boulardii group was better than in the control group (Table 4).

\section{Adverse reactions}

No serious adverse reactions occurred in any of the groups. In the control group, there was one case of constipation, which disappeared after drug withdrawal. There were no 


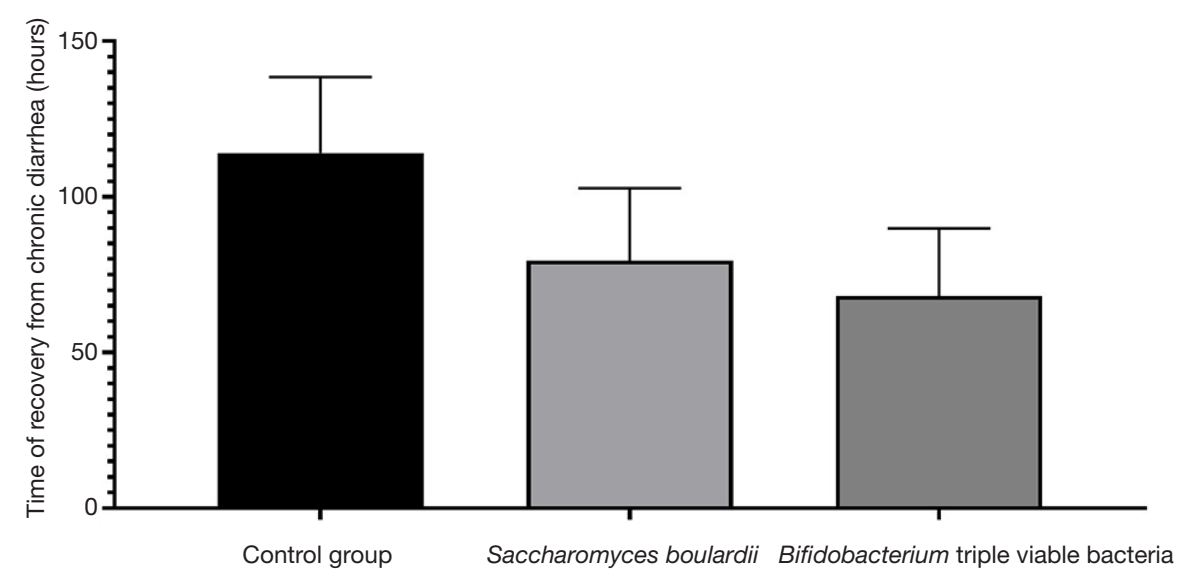

Figure 2 Time of recovery from diarrhea in conventional treatment, Saccharomyces boulardii and Bifidobacterium triple viable bacteria groups.

Table 3 Comparison of time to recovery after treatment in three groups $\left(\bar{x}_{ \pm s}\right)$

\begin{tabular}{lcccc}
\hline Group & Cases $(\mathrm{n})$ & Time to recovery $(\mathrm{h})$ & Max. (h) & Min. (h) \\
\hline Control group & 50 & $114.04 \pm 24.43^{\star \#}$ & 753 & 131 \\
Saccharomyces boulardii group & 55 & $55.51 \pm 23.25^{\#}$ & 114 & 43 \\
Bifidobacterium triple viable group & $55.13 \pm 21.71$ & 22 \\
\hline
\end{tabular}

*, $\mathrm{P}<0.05$ vs. the effect of $\mathrm{S}$. boulardii group. ", $\mathrm{P}<0.05$ vs. the effect of Bifidobacterium triple viable group.

Table 4 Changes in inflammatory factors (IL-6, IL-17, TNF- $\alpha$ ) of patients in three groups before and after treatment $\left(\bar{x}_{ \pm \mathrm{s}}\right)$

\begin{tabular}{lccccc}
\hline Group & & Cases $(\mathrm{n})$ & $\mathrm{IL}-6(\mathrm{pg} / \mathrm{mL})$ & $\mathrm{IL}-17(\mathrm{pg} / \mathrm{mL})$ & $\mathrm{TNF}-\alpha(\mathrm{pg} / \mathrm{mL})$ \\
\hline Control group & Before treatment & 50 & $26.04 \pm 3.30$ & $28.84 \pm 5.30$ & $20.02 \pm 3.89$ \\
& After treatment & 50 & $15.26 \pm 3.40^{* \# \mathbf{\Delta}}$ & $16.95 \pm 2.77^{* \# \mathbf{\Delta}}$ & $9.90 \pm 2.42^{\star \mathbf{\Delta}}$ \\
Saccharomyces boulardii & Before treatment & 55 & $28.123 \pm 3.70$ & $28.67 \pm 5.00$ & $20.66 \pm 3.85$ \\
group & After treatment & 55 & $12.09 \pm 2.08^{* \mathbf{\Delta}}$ & $13.15 \pm 1.36^{\star \mathbf{\Delta}}$ & $8.96 \pm 1.64^{*}$ \\
$\begin{array}{l}\text { Bifidobacterium triple } \\
\text { viable group }\end{array}$ & Before treatment & 55 & $27.33 \pm 3.41$ & $27.95 \pm 4.50$ & $20.86 \pm 3.56$ \\
\hline
\end{tabular}

${ }^{*}, \mathrm{P}<0.05$ vs. the levels before treatment. ${ }^{*}, \mathrm{P}<0.05$ vs. the levels of $\mathrm{S}$. boulardii group. ${ }^{\mathbf{}}, \mathrm{P}<0.05$ vs. the levels of Bifidobacterium triple viable group.

significant differences in routine blood, liver and kidney function tests in the three groups after treatment $(\mathrm{P}>0.05)$.

\section{Discussion}

Previous studies of probiotics in the treatment of diarrhea have mostly focused on acute diarrhea, viral diarrhea or AAD, and many have confirmed the effectiveness of probiotics for diarrhea symptoms in these cases (17-20). There are many reasons for CD in children in the clinic. In order to investigate whether probiotics are effective for all causes of diarrhea, this study did not limit the cause of CD, but used symptoms as an inclusion criterion. A total of 161 cases of $\mathrm{CD}$ with various causes were included. The results showed that probiotics had a better therapeutic effect on the overall symptoms of diarrhea. There were differences 
in the number of cases of different causes, and because the number of cases of some causes was too few, this study was unable to group by cause to conduct a comparative analysis.

The pathogenesis of various diseases that cause CD mostly involves the participation of dysbacterial factors. Long-term CD itself can lead to obvious intestinal flora imbalance. Diarrhea and flora imbalance are mutual cause and effect. Studies have shown that by excluding bacterial infection and repeated use of a variety of antibiotics, the number of Escherichia coli, Bacteroides spp. and Bifidobacteria in the intestines and feces of patients with CD decreased, the number of Clostridium perfringens increased, and Proteus mirabilis, Serratia liquefaciens and Enterococcus faecium became the predominant flora in the intestines, which led to changes in the intestinal microecological environment and aggravated diarrhea symptoms $(21,22)$. Therefore, all kinds of CD can be treated with probiotics. At present, studies have also confirmed that probiotics have a good effect on irritable bowel disease and intestinal allergic diseases $(23,24)$.

When infantile diarrhea occurs, the rapid reproduction of harmful flora in the gastrointestinal tract leads to an imbalance of the intestinal flora, which can lead to protracted diarrhea (25). Bifidobacterium triple viable is a type of biological agent of intestinal flora, which together with other anaerobic bacteria occupy the surface of intestinal mucosa, form a biological barrier, prevent the colonization and invasion of bacteria, produce lactic acid and acetic acid, reduce the intestinal $\mathrm{pH}$ value, inhibit the growth of pathogenic bacteria, improve the intestinal environment, and reconstruct the normal intestinal microecosystem. At the same time, Bifidobacterium can inhibit some pathogenic bacteria in children's intestines, correct the imbalance of intestinal flora, promote intestinal peristalsis, and play an antidiarrheal role (26). The digestive system of young children is not fully developed and mature, and the level of digestive enzymes and gastric acid released by the gastrointestinal tract is low, which leads to a decline in the body's resistance and reduces its immune function. Relevant studies (27) have shown that low immune function is an important factor leading to the occurrence and prolongation of diarrhea in children. Strengthening the immune function of the body can promote the control of diarrhea symptoms. Live bifidobacteria can ferment sugars, restore intestinal peristalsis, promote the synthesis of multiple vitamins and biological enzymes, improve the body's absorption of calcium, iron, vitamins and other trace elements, and enhance human immune function. The results of this study showed that the triple live bacteria of S. boulardii and Bifidobacterium were significantly better than the control treatment in terms of cure time, effect and total effective rate, and that Bifidobacterium triple viable treatment effect was superior to $S$. boulardii, suggesting that Bifidobacterium triple viable treatment of infantile diarrhea can promote improvement of symptoms and signs, and improve clinical efficacy. Focal inflammatory response is one of the important mechanisms of the occurrence and development of infantile diarrhea. Its occurrence and development are closely related to IL-6, IL-17, TNF- $\alpha$ and other inflammatory factors. Cytokines play an important role in the occurrence and development of enteritis. IL-6 is a lymphokine secreted by $\mathrm{T}$ lymphocytes, and promotes the secretion of $\mathrm{C}$-reactive protein, induces the production of acute reactive protein and aggravates the degree of inflammation (24). IL-17 is a cytokine that plays a resistance role in intestinal infection. It can recruit neutrophils at the inflammatory reaction site and play a mediating role against infection. Once children's intestinal flora is in disorder, coupled with low resistance, IL-17 will play a mediating role in intestinal immune and inflammatory reactions, leading to a sharp rise in its level (28). TNF- $\alpha$ is an inflammatory factor secreted by monocyte macrophages, which participates in the immune response and promotes the production of other inflammatory factors (29). Our results showed that after 14 days of treatment, the serum levels of IL-6, IL-17 and TNF- $\alpha$ in the Bifidobacterium triple viable group were significantly lower than those in the control group, indicating that Bifidobacterium triple viable can significantly reduce the inflammatory reaction, improve the intestinal mucosal barrier function and improve the intestinal flora distribution in children with CD. The intestinal mucosal barrier is an important part of the intestinal defense mechanism, which can prevent pathogens, endotoxin and other harmful substances from entering human tissues and blood circulation, and avoid the aggravation of injury (30).

S. boulardii also had a therapeutic effect on CD, but it was not as obvious as that of Bifidobacterium triple viable. In this study the children have good tolerance to $S$. boulardii and Bifidobacterium triple viable, and there are no related adverse reactions.

In conclusion, the treatment of $\mathrm{CD}$ in children with Bifidobacterium triple viable can significantly reduce the serum levels of IL-6, IL-17 and TNF- $\alpha$, shorten the clinical course and improve the clinical total effective rate, making 
it suitable for clinical use.

\section{Acknowledgments}

Funding: None.

\section{Footnote}

Reporting Checklist: The authors have completed the CONSORT reporting checklist. Available at http://dx.doi. org/10.21037/tp-21-195

Trial Protocol: Available at http://dx.doi.org/10.21037/tp-21-195

Data Sharing Statement: Available at http://dx.doi. org/10.21037/tp-21-195

Conflicts of Interest: All authors have completed the ICMJE uniform disclosure form (available at http://dx.doi. org/10.21037/tp-21-195). The authors have no conflicts of interest to declare.

Ethical Statement: The authors are accountable for all aspects of the work in ensuring that questions related to the accuracy or integrity of any part of the work are appropriately investigated and resolved. This study was conducted in accordance with the ethical principles of the Declaration of Helsinki (as revised in 2013), and has been approved by the ethics committee of Zhongda Hospital Affiliated to Southeast University (No. 20181092). The parents of all study subjects who met the criteria for inclusion gave informed consent.

Open Access Statement: This is an Open Access article distributed in accordance with the Creative Commons Attribution-NonCommercial-NoDerivs 4.0 International License (CC BY-NC-ND 4.0), which permits the noncommercial replication and distribution of the article with the strict proviso that no changes or edits are made and the original work is properly cited (including links to both the formal publication through the relevant DOI and the license). See: https://creativecommons.org/licenses/by-nc-nd/4.0/.

\section{References}

1. Troeger C, Blacker BF, Khalil IA, et al. Estimates of the global, regional, and national morbidity, mortality, and aetiologies of diarrhoea in 195 countries: a systematic analysis for the Global Burden of Disease Study 2016. Lancet Infect Dis 2018;18:1211-28.

2. Bhutta ZA, Ghishan F, Lindley K, et al. Persistent and chronic diarrhea and malabsorption: Working Group report of the second World Congress of Pediatric Gastroenterology, Hepatology, and Nutrition. J Pediatr Gastroenterol Nutr 2004;39 Suppl 2:S711-6.

3. Scharf RJ, Deboer MD, Guerrant RL. Recent advances in understanding the long-term sequelae of childhood infectious diarrhea. Curr Infect Dis Rep 2014;16:408.

4. Guarino A, Lo Vecchio A, Berni Canani R. Chronic diarrhoea in children. Best Pract Res Clin Gastroenterol 2012;26:649-61.

5. Zella GC, Israel EJ. Chronic diarrhea in children. Pediatr Rev 2012;33:207-17; quiz 217-8.

6. Collinson S, Deans A, Padua-Zamora A, et al. Probiotics for treating acute infectious diarrhoea. Cochrane Database Syst Rev 2020;12:CD003048.

7. Czerucka D, Piche T, Rampal P. Review article: yeast as probiotics -- Saccharomyces boulardii. Aliment Pharmacol Ther 2007;26:767-78.

8. Vandenplas Y, Brunser O, Szajewska H. Saccharomyces boulardii in childhood. Eur J Pediatr 2009;168:253-65.

9. Okubo Y, Miyairi I, Michihata N, et al. Recent Prescription Patterns for Children With Acute Infectious Diarrhea. J Pediatr Gastroenterol Nutr 2019;68:13-6.

10. Wang Y, Zhang TP, Xiao HL, et al. Formulation of an early warning infectivity score system for adult patients with acute bacterial diarrhea. Biomed Environ Sci 2014;27:65-9.

11. Xia YL, Chen HW, Wang YC, et al. Effects of microecological regulators on serum inflammatory factors, intestinal mucosal barrier function and intestinal flora in patients with acute infectious diarrhea. Chinese Journal of Nosocomial Infection 2016; 26:4844-6.

12. Zhang K, Shi L, Tang HJ, et al. Effect of Shen Song Yang Xin Capsule on Myocardial Electrophysiology of Acute Atrial Fibrillation in Canine Model. Chin Med J (Engl) 2017;130:2513-4.

13. Wang G, Feng D. Therapeutic effect of Saccharomyces boulardii combined with Bifidobacterium and on cellular immune function in children with acute diarrhea. Exp Ther Med 2019;18:2653-9.

14. Cagan E, Ceylan S, Mengi S, et al. Evaluation of Gelatin Tannate Against Symptoms of Acute Diarrhea in Pediatric Patients. Med Sci Monit 2017;23:2029-34.

15. Lin WZ, Li SC, Deng L. Differences of intestinal microflora distribution in patients with different types 
of irritable bowel syndrome and intervention effect of intestinal microecological agents. Chinese Primary Medicine 2017;24:3364-8.

16. Diagnosis and treatment of diarrhea in China. Chinese Journal of Practical Pediatrics 1998(06):61-4.

17. Wang XB. Comparison of the efficacy of Saccharomyces boulardii and Bifidobacterium Tetravaccine in the treatment of infantile rotavirus gastroenteritis. Journal of Clinical Medical Literature 2017;4:1946-7.

18. Cui S, Hu Y. Multistrain probiotic preparation significantly reduces symptoms of irritable bowel syndrome in a double-blind placebo-controlled study. Int J Clin Exp Med 2012;5:238-44.

19. Mourey F, Sureja V, Kheni D, et al. A Multicenter, Randomized, Double-blind, Placebo-controlled Trial of Saccharomyces boulardii in Infants and Children With Acute Diarrhea. Pediatr Infect Dis J 2020;39:e347-e51.

20. Choi CH, Jo SY, Park HJ, et al. A randomized, double-blind, placebo-controlled multicenter trial of saccharomyces boulardii in irritable bowel syndrome: effect on quality of life. J Clin Gastroenterol 2011;45:679-83.

21. Wu Q, Wang H. Chronic diarrhea and intestinal flora imbalance. Chinese Journal of General Practitioners 2018:778-80.

22. Li Y. Chronic diarrhea and intestinal flora imbalance. Chinese Journal of Practical Internal Medicine 2003:584-6.

23. Chitapanarux I, Chitapanarux T, Traisathit P, et al. Randomized controlled trial of live lactobacillus acidophilus plus bifidobacterium bifidum in prophylaxis of diarrhea during radiotherapy in cervical cancer patients. Radiat Oncol 2010;5:31.

24. Sanders ME, Guarner F, Guerrant R, et al. An update on the use and investigation of probiotics in health and disease. Gut 2013;62:787-96.

25. The HC, Florez de Sessions P, Jie S, et al. Assessing gut microbiota perturbations during the early phase of infectious diarrhea in Vietnamese children. Gut Microbes 2018;9:38-54.

26. Yin HY, Guo AP. Clinical observation of Bifidobacterium triple viable powder combined with Xingpi Yanger granule in the treatment of infantile pneumonia secondary diarrhea. Modern Medicine and Clinical 2017:1921-4.

27. Wang JH, Liu L, Sun ZH, et al. Efficacy of Jianpi Zhixie decoction combined with massage in the treatment of infantile diarrhea and its influence on immune function. Chinese Journal of traditional Chinese Medicine 2019:400-2.

28. Tao L, Tu ZL, Wang P, et al. Application of Bifidobacterium Tetravaccine in prevention of nosocomial infection. Chinese Journal of Nosocomial Infection 2013;23:5402-3+6.

29. He X, Cui LH, Wang XH, et al. Modulation of inflammation by toll-like receptor 4/nuclear factor-kappa $\mathrm{B}$ in diarrhea-predominant irritable bowel syndrome. Oncotarget 2017;8:113957-65.

30. Shi YC, Yang YS. Microecological agents and gastrointestinal diseases. Chinese Journal of Practical Internal Medicine 2016;36:729-32.

(English Language Editor: K. Brown)
Cite this article as: Zhang J, Wan S, Gui Q. Comparison of safety, effectiveness and serum inflammatory factor indexes of Saccharomyces boulardii versus Bifidobacterium triple viable in treating children with chronic diarrhea: a randomized trial. Transl Pediatr 2021;10(6):1677-1685. doi: 10.21037/tp-21-195 